\title{
AN HI STUDY OF URSA MAJOR SPIRALS.
}

Dark Matter in spirals and the TF-relations

\author{
M.A.W. VERHEIJEN \\ Kapteyn Institute \\ Postbus 800, 9700 AV Groningen, The Netherlands
}

We investigate the scatter in the TF-relations and study the Dark Matter component of spiral galaxies as a function of luminosity, morphology, scale length etc. by means of a detailed kinematic and photometric study of individual galaxies in a cluster. Because all the galaxies are at the same distance there is no doubt about their relative masses and luminosities.

A galaxy is considered to be a member of the Ursa Major cluster if its position on the sky $<7.5$ degrees from $\alpha=11^{h} 56^{m} \cdot 9 \delta=49^{d} 22^{\prime}$ and $700<V_{\text {sys }}<1210 \mathrm{kms}^{-1}$. So far, 79 galaxies are identified as cluster members. The currently forming cluster is rich in spirals and has a low velocity dispersion of $\approx 150 \mathrm{kms}^{-1}$. The estimated depth of the cluster contributes $\approx 0^{m} .17$ to the scatter in the TF-relations.

B, R, I and K' surface photometry obtained with the UH 24 " and 88 " telescopes on Mauna Kea is available for 78 galaxies. Analysis of the luminosity profiles is currently in progress. $\mathrm{HI} 21 \mathrm{~cm}$-line synthesis observations done with the WSRT provide HI line widths and rotation curves for 62 observed galaxies of which 32 galaxies are reduced and analyzed.

The cluster environment does not seem to influence the HI properties of the spirals as is the case in Virgo (Cayatte et al, 1994). Of the 32 galaxies analyzed so far, 19 have published H-band magnitudes (Tormen and Burstein, 1995). Using the maximum rotational velocities from the 19 rotation curves, the biweight (Beers et al, 1990) scatter in the TF-relation after an inverse fit is $0^{m} .26$ while using the amplitude of the last measured point on the rotation curve results in a biweight scatter of $0^{m} .40$. A quadrature subtraction of $0^{m} .17$ from $0^{m} .26$ results in a observational plus intrinsic scatter of $0^{m} .20$ or a $9 \%$ uncertainty in distance.

\section{References}

Beers, T.C., Flynn, K. and Gebhardt, K. (1990), A.J., 100, p. 32

Cayatte, V., Kotanyi, C., Balkowski, C. and van Gorkom, J.H. (1994), A.J., 107, p. 1003

Tormen, G. and Burstein, D. (1995), Ap.J.Suppl., 96, p. 123 